\title{
An approximation of a common fixed point of nonexpansive mappings on convex metric spaces
}

\author{
W Anakkamatee and S Dhompongsa*
}

\section{"Correspondence:}

sompongd@chiangmai.ac.th Department of Mathematics, Faculty of Science, Chiang Mai University, Chiang Mai, 50200, Thailand

\begin{abstract}
Sokhuma and Kaewkhao (2011) introduced an iteration scheme to compute a common fixed point of a single-valued nonexpansive mapping and a multivalued nonexpansive mapping on a uniformly convex Banach space. In this paper, we extend the above result of Sokhuma and Kaewkhao from a single-valued mapping to a countable number of mappings and, at the same time, we extend the underlying spaces to strictly convex Banach spaces. The corresponding results are also obtained for the CAT(0) space setting.
\end{abstract}

MSC: $47 \mathrm{H} 09 ; 47 \mathrm{H} 10$

Keywords: common fixed point; nonexpansive mapping; strictly convex Banach space; CAT(0) space

\section{Introduction}

Let $X$ be a complete metric space, and $E$ a nonempty subset of $X$. We will denote by $2^{E}$ the family of nonempty subsets of $E$ and by $F B(E)$ the family of nonempty bounded closed subsets of $E$. Let $H(\cdot, \cdot)$ be theHausdorff distance on $F B(X)$, that is,

$$
H(A, B)=\max \left\{\sup _{a \in A} \operatorname{dist}(a, B), \sup _{b \in B} \operatorname{dist}(b, A)\right\}, \quad A, B \in F B(X),
$$

where $\operatorname{dist}(a, B)=\inf \{d(a, b): b \in B\}$ is the distance from the point $a$ to the subset $B$.

A mapping $t: E \rightarrow E$ and a multivalued mapping $T: E \rightarrow F B(X)$ are said to be nonexpansive if for each $x, y \in E$,

$$
\begin{aligned}
& d(t x, t y) \leq d(x, y), \quad \text { and } \\
& H(T x, T y) \leq d(x, y),
\end{aligned}
$$

respectively. If $t x=x$, we call $x$ a fixed point of a single-valued mapping $t$. Moreover, if $x \in T x$, we call $x$ a fixed point of a multivalued mapping $T$. We use the notation $\operatorname{Fix}(S)$ to stand for the set of fixed points of a mapping $S$. Thus $\operatorname{Fix}(t) \cap \operatorname{Fix}(T)$ is the set of common fixed points of $t$ and $T$, i.e., $x \in \operatorname{Fix}(t) \cap \operatorname{Fix}(T)$ if and only if $x=t x \in T x$.

Following [8], a bounded closed and convex subset $E$ of a Banach space $X$ has the fixed point property for nonexpansive mappings (FPP) (respectively, for multivalued nonexpansive mappings (MFPP)) if every nonexpansive mapping of $E$ into $E$ has a fixed point 
(respectively, every nonexpansive mapping of $E$ into $2^{E}$ with compact convex values has a fixed point). For a bounded closed and convex subset $E$ of a Banach space $X$, a mapping $t: E \rightarrow X$ is said to satisfy the conditional fixed point property (CFP) if either $t$ has no fixed points, or $t$ has a fixed point in each nonempty bounded closed convex set that leaves $t$ invariant. A set $E$ is said to have the conditional fixed point property for nonexpansive mappings (CFPP) if every nonexpansive $t: E \rightarrow E$ satisfies (CFP). For commuting family of nonexpansive mappings, the following is a remarkable common fixed point property due to Bruck [6].

Theorem 1.1 ([6]) Let X be a Banach space and E a nonempty closed convex subset of X. If $E$ has both the (FPP) and the (CFPP) for nonexpansive mappings, then for any commuting family $\mathcal{S}$ of nonexpansive mappings of $E$ into $E$, there is a common fixed point for $\mathcal{S}$.

Theorem 1.1 was proved by Belluce and Kirk [1] when $\mathcal{S}$ is finite and $E$ is weakly compact and has a normal structure; by Belluce and Kirk [2] when $E$ is weakly compact and has a complete normal structure; by Browder [4] when $X$ is uniformly convex and $E$ is bounded; by Lau and Holmes [11] when $\mathcal{S}$ is left reversible and $E$ is compact; and finally, by Lim [14] when $\mathcal{S}$ is left reversible and $E$ is weakly compact and has a normal structure.

Open Problem (Bruck [6]). Can commutativity of $\mathcal{S}$ be replaced by left reversibility?

The answer to this Problem is not known even when the semigroup is left amenable (see [13] for more details).

In 2011, Sokhuma and Kaewkhao [17] introduced a new iteration method for approximating a common fixed point of a pair of a single-valued and a multivalued nonexpansive mappings and proved the following strong convergence theorem:

Theorem 1.2 ([17, Theorem 3.5]) Let E be a nonempty compact convex subset of a uniformly convex Banach space $X$, and let $t: E \rightarrow E$ and $T: E \rightarrow F B(E)$ be a single-valued and a multivalued nonexpansive mappings respectively, and $\operatorname{Fix}(t) \cap \operatorname{Fix}(T) \neq \emptyset$ satisfying $T w=\{w\}$ for all $w \in \operatorname{Fix}(t) \cap \operatorname{Fix}(T)$. Let $\left\{x_{n}\right\}$ be the sequence of the modified Ishikawa iteration defined by

$$
\begin{aligned}
& y_{n}=\left(1-\beta_{n}\right) x_{n}+\beta_{n} z_{n}, \\
& x_{n+1}=\left(1-\alpha_{n}\right) x_{n}+\alpha_{n} t y_{n},
\end{aligned}
$$

where $x_{1} \in E, z_{n} \in T x_{n}$ and $0<a \leq \alpha_{n}, \beta_{n} \leq b<1$. Then $\left\{x_{n}\right\}$ converges strongly to a common fixed point of $t$ and $T$.

For a single-valued nonexpansive mapping $t: E \rightarrow E$ with $\operatorname{Fix}(t) \neq \emptyset$, where $E$ is a convex nonexpansive retract of a real uniformly smooth Banach space, Reich and Shemen [15, Theorem 3.4] obtained a strong convergence to a fixed point of $t$ of a sequence $\left\{x_{n}\right\}$ of the form

$$
\begin{aligned}
& y_{n}=R_{E}\left[\left(1-\beta_{n}\right) x_{n}\right], \\
& x_{n+1}=\left(1-\alpha_{n}\right) x_{n}+\alpha_{n} t y_{n},
\end{aligned}
$$

where $R_{E}$ is a retraction on the subset $E$ and the sequences $\left\{\alpha_{n}\right\},\left\{\beta_{n}\right\}$ satisfy conditions: (i) $0<\liminf _{n \rightarrow \infty} \alpha_{n} \leq \limsup _{n \rightarrow \infty} \alpha_{n}<1$, (ii) $\lim _{n \rightarrow \infty} \beta_{n}=0$ and $\sum_{n=1}^{\infty} \beta_{n}=\infty$. Clearly, conditions (i) and (ii) on the sequences $\left\{\alpha_{n}\right\},\left\{\beta_{n}\right\}$ are different from the ones in Theorem 1.2. 
In 2003, Suzuki [18] proved the following result.

Theorem 1.3 ([18, Theorem 2]) Let E be a compact convex subset of a strictly convex Banach space $X$. Let $\left\{t_{n}: n \in \mathbb{N}\right\}$ be a sequence of nonexpansive mappings on $E$ with $\bigcap_{n=1}^{\infty} \operatorname{Fix}\left(t_{n}\right) \neq \emptyset$. Let $\left\{\gamma_{n}\right\}$ be a sequence of positive numbers such that $\sum_{n=1}^{\infty} \gamma_{n}<1$, and let $\left\{I_{n}\right\}$ be a sequence of subsets of $\mathbb{N}$ satisfying $I_{n} \subset I_{n+1}$ for $n \in \mathbb{N}$ and $\bigcup_{n=1}^{\infty} I_{n}=\mathbb{N}$. Define a sequence $\left\{x_{n}\right\}$ in $E$ by $x_{1} \in C$ and

$$
x_{n+1}=\left(1-\sum_{i \in I_{n}} \gamma_{i}\right) x_{n}+\sum_{i \in I_{n}} \gamma_{i} t_{i} x_{n}
$$

for $n \in \mathbb{N}$. Then $\left\{x_{n}\right\}$ converges strongly to a common fixed point of $\left\{t_{n}: n \in \mathbb{N}\right\}$.

The purpose of this paper is to extend Theorem 1.2 to countably many numbers of single-valued nonexpansive mappings on strictly convex Banach spaces, thereby the result in Theorem 1.3 is covered. The results for $\mathrm{CAT}(0)$ spaces are also derived. Our main discoveries are Theorem 3.2 and Theorem 3.6.

\section{Preliminaries}

We recall that the graph $G(U)$ of a multivalued mapping $U: E \rightarrow 2^{X}$ is $G(U)=\{(x, y) \in$ $X \times X ; x \in E, y \in U x\}$. The following theorem is essentially proved by Dozo [10].

Theorem 2.1 ([10, Theorem 3.1]) Let $X$ be a Banach space which satisfies Opial's condition, $E$ be a weakly compact convex subset of $X$. Let $T: E \rightarrow K(X)$, where $K(X)$ is a family of nonempty compact subsets of $X$. Then the graph of $U=I-T$ is closed in $\left(X, \sigma\left(X, X^{*}\right)\right) \times(X,\|\cdot\|)$, where I denotes the identity on $X, \sigma\left(X, X^{*}\right)$ the weak topology and $\|\cdot\|$ the norm (or strong) topology.

We will use the theorem in the following form: If $\left\{x_{n}\right\}$ is a sequence in $E$ such that $\left\{x_{n}\right\}$ converges weakly to some $z \in E$ and $\left\{\operatorname{dist}\left(x_{n}, T x_{n}\right)\right\}$ converges to 0 , then $z \in T z$.

Let $\left\{t_{n}: n \in \mathbb{N}\right\}$ be a family of nonexpansive mappings from $E$ to $E$. The following lemma proved by Bruck [5] plays a very important role to our proof of the main result.

Lemma 2.2 ([5, Lemma 3]) Let E be a nonempty closed convex subset of a strictly convex Banach space $X$, let $\left\{t_{n}: n \in \mathbb{N}\right\}$ be a family of single-valued nonexpansive mappings on E. Suppose $\bigcap_{n=1}^{\infty} \operatorname{Fix}\left(t_{n}\right)$ is nonempty. Given $\left\{\lambda_{n}\right\}$ a sequence of positive numbers with $\sum_{n=1}^{\infty} \lambda_{n}=1$. Then a mapping $t$ on $E$ defined by

$$
t x=\sum_{n=1}^{\infty} \lambda_{n} t_{n} x
$$

for all $x \in E$ is well defined, nonexpansive and $\operatorname{Fix}(t)=\bigcap_{n=1}^{\infty} \operatorname{Fix}\left(t_{n}\right)$.

The following results show examples when the required condition on the nonemptiness of the common fixed point set always satisfies:

Theorem 2.3 ([8, Theorem 3.1]) Let E be a weakly compact convex subset of a Banach space X. Suppose E has (MFPP) and (CFPP). Let $\mathcal{S}$ be any commuting family of nonexpansive self-mappings of $E$. If $T: E \rightarrow K C(E)$ is a multivalued nonexpansive mapping which 
commutes with every member of $\mathcal{S}$, where $K C(E)$ is the family of nonempty compact convex subsets of $E$. Then $F(\mathcal{S}) \cap \operatorname{Fix}(T) \neq \emptyset$ where $F(\mathcal{S})=\bigcap_{t \in \mathcal{S}} \operatorname{Fix}(t)$.

Theorem 2.4 ([8, Theorem 3.2]) Let X be a Banach space satisfying the Kirk-Massa condition, i.e., the asymptotic center of each bounded sequence of $X$ in each bounded closed and convex subset is nonempty and compact. Let $E$ be a weakly compact convex subset of $X$ and let $\mathcal{S}$ be any commuting family of nonexpansive self-mappings of E. Suppose $T: E \rightarrow K C(E)$ is a multivalued mapping satisfying condition $\left(C_{\lambda}\right)$ for some $\lambda \in(0,1)$ which commutes with every member of $\mathcal{S}$. If $T$ is upper semi-continuous, then $F(\mathcal{S}) \cap \operatorname{Fix}(T) \neq \emptyset$.

Note that strictly convex Banach spaces satisfy the condition in the above theorems.

Remark 2.5 In our main theorems (Theorem 3.2 and Theorem 3.6), we assume the following conditions:

$$
F(\mathcal{S}) \cap \operatorname{Fix}(T) \neq \emptyset \quad \text { and } \quad T w=\{w\} \quad \text { for all } w \in F(\mathcal{S}) \cap \operatorname{Fix}(T) .
$$

It is an open problem to find a sufficient condition to assure that the condition (2.1) is satisfied.

Let $(X, d)$ be a metric space. A geodesic joining $x \in X$ to $y \in X$ is a mapping $c$ from a closed interval $[0, l] \subset \mathbb{R}$ to $X$ such that $c(0)=x, c(l)=y$ and $d\left(c(t), c\left(t^{\prime}\right)\right)=\left|t-t^{\prime}\right|$ for all $t, t^{\prime} \in[0, l]$. Thus $c$ is an isometry and $d(x, y)=l$. The image of $c$ is called a geodesic (or metric) segment joining $x$ and $y$. We denote $[x, y]$ for this geodesic if it is unique. Write $c(\alpha 0+(1-\alpha) l)=\alpha x \oplus(1-\alpha) y$ for $\alpha \in(0,1)$. The space $X$ is said to be a geodesic space if every two points of $X$ are joined by a geodesic. It is said to be of hyperbolic type [12] if it satisfies:

$$
d(p, \alpha x \oplus(1-\alpha) y) \leq \alpha d(p, x)+(1-\alpha) d(p, y)
$$

for all $p \in X$. Let $\left\{v_{1}, v_{2}, \ldots, v_{n}\right\} \subset X$ and $\left\{\lambda_{1}, \lambda_{2}, \ldots, \lambda_{n}\right\} \subset(0,1)$ with $\sum_{i=1}^{n} \lambda_{i}=1$. It had been defined, by induction, in [7] that

$$
\bigoplus_{i=1}^{n} \lambda_{i} v_{i}:=\left(1-\lambda_{n}\right)\left(\frac{\lambda_{1}}{1-\lambda_{n}} v_{1} \oplus \frac{\lambda_{2}}{1-\lambda_{n}} v_{2} \oplus \cdots \oplus \frac{\lambda_{n-1}}{1-\lambda_{n}} v_{n-1}\right) \oplus \lambda_{n} v_{n} .
$$

The definition of $\oplus$ in (2.3) is an ordered one in the sense that it depends on the order of points $v_{1}, \ldots, v_{n}$. Under (2.2) we can see that

$$
d\left(\bigoplus_{i=1}^{n} \lambda_{i} v_{i}, x\right) \leq \sum_{n=1}^{n} \lambda_{i} d\left(v_{i}, x\right)
$$

for each $x \in X$.

Following [3], a metric space $X$ is said to be a CAT(0) space if it is geodesically connected and if every geodesic triangle in $X$ is at least as thin as its comparison triangle in the Euclidean plane $\mathbb{E}^{2}$. In fact (cf. [3] p.163), the following are equivalent for a geodesic space $X$ :

(i) $X$ is a $\mathrm{CAT}(0)$ space. 
(ii) $X$ satisfies the $(\mathrm{CN})$ inequality: If $x_{0}, x_{1} \in X$ and $\frac{x_{0} \oplus x_{1}}{2}$ is the midpoint of $x_{0}$ and $x_{1}$, then

$$
d^{2}\left(y, \frac{x_{0} \oplus x_{1}}{2}\right) \leq \frac{1}{2} d^{2}\left(y, x_{0}\right)+\frac{1}{2} d^{2}\left(y, x_{1}\right)-\frac{1}{4} d^{2}\left(x_{0}, x_{1}\right), \quad \text { for all } y \in X
$$

Lemma 2.6 ([3, Proposition 2.2]) Let $X$ be a CAT(0) space. Then for each $p, q, r, s \in X$ and $\alpha \in[0,1]$,

$$
d(\alpha p \oplus(1-\alpha) q, \alpha r \oplus(1-\alpha) s) \leq \alpha d(p, r)+(1-\alpha) d(q, s) .
$$

In particular, (2.2) holds in $\mathrm{CAT}(0)$ spaces.

In [9] the element $x=\bigoplus_{n=1}^{\infty} \lambda_{n} v_{n}$ has been defined. Let $\left\{\lambda_{n}\right\}$ be a given sequence in $(0,1)$ such that $\sum_{n=1}^{\infty} \lambda_{n}=1$, let $\left\{v_{n}\right\}$ be a bounded sequence in $X$, and let $v_{0}$ be an arbitrary point in $X$. Let $\lambda_{n}^{\prime}=\sum_{i=n+1}^{\infty} \lambda_{i}$ and assume that $\sum_{i=n}^{\infty} \lambda_{i}^{\prime} \rightarrow 0$ as $n \rightarrow \infty$. Set

$$
s_{n}:=\lambda_{1} v_{1} \oplus \lambda_{2} v_{2} \oplus \cdots \oplus \lambda_{n} v_{n} \oplus \lambda_{n}^{\prime} v_{0}
$$

Thus, by (2.3),

$$
s_{n}=\left(\sum_{i=1}^{n} \lambda_{i}\right) w_{n} \oplus \lambda_{n}^{\prime} v_{0}
$$

where $w_{1}=v_{1}$ and for each $n \geq 2$,

$$
w_{n}=\frac{\lambda_{1}}{\sum_{i=1}^{n} \lambda_{i}} v_{1} \oplus \frac{\lambda_{2}}{\sum_{i=1}^{n} \lambda_{i}} v_{2} \oplus \cdots \oplus \frac{\lambda_{n}}{\sum_{i=1}^{n} \lambda_{i}} v_{n} .
$$

We know that $\left\{s_{n}\right\}$ is a Cauchy sequence (see [9]). Thus $s_{n} \rightarrow x$ as $n \rightarrow \infty$ for some $x \in X$. Write

$$
x=\bigoplus_{n=1}^{\infty} \lambda_{n} v_{n}
$$

By (2.6), $d\left(s_{n}, w_{n}\right) \leq \lambda_{n}^{\prime} d\left(w_{n}, v_{0}\right)$, it is seen that $\lim _{n \rightarrow \infty} s_{n}=\lim _{n \rightarrow \infty} w_{n}$. Thus the limit $x$ is independent of the choice of $v_{0}$.

Lemma 2.7 ([9, Lemma 3.8]) Let $C$ be a nonempty closed convex subset of a complete CAT(0) space $X$, let $\left\{t_{n}: n \in \mathbb{N}\right\}$ be a family of single-valued nonexpansive mappings on $C$. Suppose $\bigcap_{n=1}^{\infty} \operatorname{Fix}\left(t_{n}\right)$ is nonempty. Define $t: C \rightarrow C$ by $t(x)=\bigoplus_{n=1}^{\infty} \lambda_{n} t_{n}(x)$ for all $x \in C$ where $\left\{\lambda_{n}\right\} \subset(0,1)$ with $\sum_{n=1}^{\infty} \lambda_{n}=1$ and $\sum_{i=n}^{\infty} \lambda_{i}^{\prime} \rightarrow 0$ as $n \rightarrow \infty$. Then $t$ is nonexpansive and $\operatorname{Fix}(t)=\bigcap_{n=1}^{\infty} \operatorname{Fix}\left(t_{n}\right)$.

\section{Main results}

\subsection{Strictly convex Banach spaces}

The following result is a generalization of the result of [16, Lemma 1.3]. 
Lemma 3.1 Let $E$ be a compact subset of a strictly convex Banach space $X$, let $\left\{\alpha_{n}\right\}$ be a sequence of real numbers such that $0<a \leq \alpha_{n} \leq b<1$ for all $n \in \mathbb{N}$, and let $\left\{u_{n}\right\},\left\{v_{n}\right\}$ be sequences of $E$ satisfying, for some $c \geq 0$,

(i) $\limsup _{n \rightarrow \infty}\left\|u_{n}\right\| \leq c$,

(ii) $\limsup _{n \rightarrow \infty}\left\|v_{n}\right\| \leq c$ and

(iii) $\lim _{n \rightarrow \infty}\left\|\alpha_{n} u_{n}+\left(1-\alpha_{n}\right) v_{n}\right\|=c$.

Then, $\lim _{n \rightarrow \infty}\left\|u_{n}-v_{n}\right\|=0$.

Proof We suppose on the contrary that $\lim \sup _{n \rightarrow \infty}\left\|u_{n}-v_{n}\right\| \neq 0$. Since $E$ and $[a, b]$ are compact, there exist subsequences $\left\{u_{n_{k}}\right\}$ of $\left\{u_{n}\right\},\left\{v_{n_{k}}\right\}$ of $\left\{v_{n}\right\}$ and $\left\{\alpha_{n_{k}}\right\}$ of $\left\{\alpha_{n}\right\}$ such that $\lim _{k \rightarrow \infty} u_{n_{k}}=u, \lim _{k \rightarrow \infty} v_{n_{k}}=v, \lim _{k \rightarrow \infty} \alpha_{n_{k}}=\alpha$ for some $u, v \in E$ with $u \neq v$ and for some $\alpha \in[0,1]$. From (i) and (ii) we have $\|u\|=\lim _{k \rightarrow \infty}\left\|u_{n_{k}}\right\| \leq c$ and $\|v\|=\lim _{k \rightarrow \infty}\left\|v_{n_{k}}\right\| \leq c$. Using the strict convexity of $X$ and (iii), we have $c=\lim _{k \rightarrow \infty}\left\|\alpha_{n_{k}} u_{n_{k}}+\left(1-\alpha_{n_{k}}\right) v_{n_{k}}\right\|=\| \alpha u+$ $(1-\alpha) v\|<\alpha\| u\|+(1-\alpha)\| v \| \leq c$, a contradiction. Hence $\lim _{n \rightarrow \infty}\left\|u_{n}-v_{n}\right\|=0$.

Now we introduce a new iteration method for a family of single-valued nonexpansive mappings and a multivalued nonexpansive mapping. Let $E$ be a nonempty bounded closed convex subset of a Banach space $X$, let $\left\{t_{n}: n \in \mathbb{N}\right\}$ be a family of single-valued nonexpansive mappings on $E$, and let $T: E \rightarrow F B(E)$ be a multivalued nonexpansive mapping. Given a sequence of positive numbers $\left\{\gamma_{n}\right\}$ with $\sum_{n=1}^{\infty} \gamma_{n}<1$. The sequence $\left\{x_{n}\right\}$ of the modified Ishikawa iteration is defined by $x_{1} \in E$, and

$$
\begin{aligned}
& y_{n}=\left(1-\beta_{n}\right) x_{n}+\beta_{n} z_{n}, \\
& x_{n+1}=\left(1-\sum_{i=1}^{n} \gamma_{i}\right) x_{n}+\sum_{i=1}^{n} \gamma_{i} t_{i} y_{n},
\end{aligned}
$$

where $z_{n} \in T x_{n}$, and $0<a \leq \beta_{n} \leq b<1$. Put $F:=\left(\bigcap_{n}^{\infty} \operatorname{Fix}\left(t_{n}\right)\right) \cap \operatorname{Fix}(T)$.

Theorem 3.2 Let $E$ be a nonempty compact convex subset of a strictly convex Banach space $X$, let $\left\{t_{n}: n \in \mathbb{N}\right\}$ be a family of single-valued nonexpansive mappings on $E$, and let $T: E \rightarrow F B(E)$ be a multivalued nonexpansive mapping. Suppose $F \neq \emptyset$ and $T w=\{w\}$ for all $w \in F$. Given a sequence of positive numbers $\left\{\gamma_{n}\right\}$ with $\sum_{n=1}^{\infty} \gamma_{n}<1$ and $\left\{\beta_{n}\right\}$ with $0<a \leq \beta_{n} \leq b<1$. Then the sequence $\left\{x_{n}\right\}$ defined by (3.1) converges strongly to some $v \in F$.

Proof We follow the proof of [17, Theorem 3.6] and split the proof into five steps.

Step 1. $\lim _{n \rightarrow \infty}\left\|x_{n}-w\right\|$ exists for all $w \in F$ :

We first note that, since $T w=\{w\}$,

$$
\left\|z_{n}-w\right\|=\operatorname{dist}\left(z_{n}, T w\right) \leq H\left(T x_{n}, T w\right) \leq\left\|x_{n}-w\right\| .
$$

Consider the following estimates:

$$
\begin{aligned}
\left\|x_{n+1}-w\right\| & \leq\left(1-\sum_{i=1}^{n} \gamma_{i}\right)\left\|x_{n}-w\right\|+\sum_{i=1}^{n} \gamma_{i}\left\|t_{i} y_{n}-w\right\| \\
& \leq\left(1-\sum_{i=1}^{n} \gamma_{i}\right)\left\|x_{n}-w\right\|+\sum_{i=1}^{n} \gamma_{i}\left\|y_{n}-w\right\|
\end{aligned}
$$




$$
\begin{aligned}
& \leq\left(1-\sum_{i=1}^{n} \gamma_{i}\right)\left\|x_{n}-w\right\|+\left(\sum_{i=1}^{n} \gamma_{i}\right)\left(\left(1-\beta_{n}\right)\left\|x_{n}-w\right\|+\beta_{n}\left\|z_{n}-w\right\|\right) \\
& \leq\left\|x_{n}-w\right\| .
\end{aligned}
$$

Therefore, $\left\{\left\|x_{n}-w\right\|\right\}$ is a bounded decreasing sequence in $\mathbb{R}$, and hence $\lim _{n \rightarrow \infty}\left\|x_{n}-w\right\|$ exists.

Step 2. $\lim _{n \rightarrow \infty}\left\|x_{n}-\frac{\sum_{i=1}^{n} \gamma_{i} t_{i} y_{n}}{\sum_{i=1}^{n} \gamma_{i}}\right\|=0$ :

From Step 1, suppose $\lim _{n \rightarrow \infty}\left\|x_{n}-w\right\|=c$. We have

$$
\begin{aligned}
\left\|\frac{\sum_{i=1}^{n} \gamma_{i} t_{i} y_{n}}{\sum_{i=1}^{n} \gamma_{i}}-w\right\| & \leq \frac{1}{\sum_{i=1}^{n} \gamma_{i}}\left\|\sum_{i=1}^{n} \gamma_{i} t_{i} y_{n}-\sum_{i=1}^{n} \gamma_{i} w\right\| \\
& \leq\left\|y_{n}-w\right\| \leq\left\|x_{n}-w\right\| .
\end{aligned}
$$

Thus

$$
\limsup _{n \rightarrow \infty}\left\|\frac{\sum_{i=1}^{n} \gamma_{i} t_{i} y_{n}}{\sum_{i=1}^{n} \gamma_{i}}-w\right\| \leq \limsup _{n \rightarrow \infty}\left\|y_{n}-w\right\| \leq \limsup _{n \rightarrow \infty}\left\|x_{n}-w\right\|=c .
$$

We also have

$$
\begin{aligned}
c & =\lim _{n \rightarrow \infty}\left\|x_{n+1}-w\right\| \\
& =\lim _{n \rightarrow \infty}\left\|\left(1-\sum_{i=1}^{n} \gamma_{i}\right) x_{n}+\sum_{i=1}^{n} \gamma_{i} t_{i} y_{n}-w\right\| \\
& =\lim _{n \rightarrow \infty}\left\|\left(1-\sum_{i=1}^{n} \gamma_{i}\right)\left(x_{n}-w\right)+\sum_{i=1}^{n} \gamma_{i}\left(\frac{\sum_{i=1}^{n} \gamma_{i} t_{i} t_{n}}{\sum_{i=1}^{n} \gamma_{i}}-w\right)\right\| .
\end{aligned}
$$

By Lemma 3.1, since $0<\gamma_{1}<\sum_{i=1}^{n} \gamma_{i} \leq \sum_{i=1}^{\infty} \gamma_{i}<1, \lim _{n \rightarrow \infty}\left\|x_{n}-\frac{\sum_{i=1}^{n} \gamma_{i} t_{i} y_{n}}{\sum_{i=1}^{n} \gamma_{i}}\right\|=\lim _{n \rightarrow \infty} \|\left(x_{n}-\right.$ $w)-\left(\frac{\sum_{i=1}^{n} \gamma_{i} t_{i} y_{n}}{\sum_{i=1}^{n} \gamma_{i}}-w\right) \|=0$.

Step 3. $\lim _{n \rightarrow \infty}\left\|x_{n}-z_{n}\right\|=0$ :

From (3.1), we can see that

$$
\left\|x_{n+1}-w\right\| \leq\left(1-\sum_{i=1}^{n} \gamma_{i}\right)\left\|x_{n}-w\right\|+\sum_{i=1}^{n} \gamma_{i}\left\|y_{n}-w\right\|,
$$

and hence $\left\|x_{n+1}-w\right\|-\left\|x_{n}-w\right\| \leq \sum_{i=1}^{n} \gamma_{i}\left(\left\|y_{n}-w\right\|-\left\|x_{n}-w\right\|\right)$. Therefore, $\left(\frac{\left\|x_{n+1}-w\right\|-\left\|x_{n}-w\right\|}{\sum_{i=1}^{n} \gamma_{i}}\right)+$ $\left\|x_{n}-w\right\| \leq\left\|y_{n}-w\right\|$ and by (3.2) we obtain

$$
\begin{aligned}
c & =\liminf _{n \rightarrow \infty}\left\{\left(\frac{\left\|x_{n+1}-w\right\|-\left\|x_{n}-w\right\|}{\sum_{i=1}^{n} \gamma_{i}}\right)+\left\|x_{n}-w\right\|\right\} \\
& \leq \liminf _{n \rightarrow \infty}\left\|y_{n}-w\right\| \leq \limsup _{n \rightarrow \infty}\left\|y_{n}-w\right\| \leq c .
\end{aligned}
$$

Thus $c=\lim _{n \rightarrow \infty}\left\|y_{n}-w\right\|=\lim _{n \rightarrow \infty}\left\|\left(1-\beta_{n}\right)\left(x_{n}-w\right)+\beta_{n}\left(z_{n}-w\right)\right\|$. By Lemma 3.1, since $0<a \leq \beta_{n} \leq b<1, \lim _{n \rightarrow \infty}\left\|x_{n}-z_{n}\right\|=0$.

Step 4. $\lim _{n \rightarrow \infty}\left\|x_{n}-\frac{\sum_{i=1}^{\infty} \gamma_{i} t_{i} x_{n}}{\sum_{i=1}^{\infty} \gamma_{i}}\right\|=0$ : 
We note from Step 3 that

$$
\left\|\frac{\sum_{i=1}^{n} \gamma_{i} t_{i} x_{n}}{\sum_{i=1}^{n} \gamma_{i}}-\frac{\sum_{i=1}^{n} \gamma_{i} t_{i} y_{n}}{\sum_{i=1}^{n} \gamma_{i}}\right\| \leq\left\|x_{n}-y_{n}\right\|=\beta_{n}\left\|x_{n}-z_{n}\right\| \rightarrow 0 \quad \text { as } n \rightarrow \infty
$$

and

$$
\begin{aligned}
\left\|t_{i} x_{n}\right\| & \leq\left\|t_{i} x_{n}-w\right\|+\|w\| \leq\left\|x_{n}-w\right\|+\|w\| \\
& \leq\left\|x_{1}-w\right\|+\|w\|:=M
\end{aligned}
$$

for all $i \in \mathbb{N}$. Therefore,

$$
\begin{aligned}
\left\|x_{n}-\frac{\sum_{i=1}^{\infty} \gamma_{i} t_{i} x_{n}}{\sum_{i=1}^{\infty} \gamma_{i}}\right\| \leq & \left\|x_{n}-\frac{\sum_{i=1}^{n} \gamma_{i} t_{i} x_{n}}{\sum_{i=1}^{n} \gamma_{i}}\right\|+\left\|\frac{\sum_{i=1}^{n} \gamma_{i} t_{i} x_{n}}{\sum_{i=1}^{n} \gamma_{i}}-\frac{\sum_{i=1}^{\infty} \gamma_{i} t_{i} x_{n}}{\sum_{i=1}^{\infty} \gamma_{i}}\right\| \\
\leq & \left\|x_{n}-\frac{\sum_{i=1}^{n} \gamma_{i} t_{i} x_{n}}{\sum_{i=1}^{n} \gamma_{i}}\right\|+\left\|\frac{\sum_{i=1}^{n} \gamma_{i} t_{i} x_{n}}{\sum_{i=1}^{n} \gamma_{i}}-\frac{\sum_{i=1}^{n} \gamma_{i} t_{i} x_{n}}{\sum_{i=1}^{\infty} \gamma_{i}}\right\| \\
& +\frac{1}{\sum_{i=1}^{\infty} \gamma_{i}} \sum_{i=n+1}^{\infty} \gamma_{i}\left\|t_{i} x_{n}\right\| \\
\leq & \left\|x_{n}-\frac{\sum_{i=1}^{n} \gamma_{i} t_{i} x_{n}}{\sum_{i=1}^{n} \gamma_{i}}\right\|+\frac{\sum_{i=n+1}^{\infty} \gamma_{i}}{\left(\sum_{i=1}^{n} \gamma_{i}\right)\left(\sum_{i=1}^{\infty} \gamma_{i}\right)} \sum_{i=1}^{n} \gamma_{i} M \\
& +\frac{\sum_{i=n+1}^{\infty} \gamma_{i}}{\sum_{i=1}^{\infty} \gamma_{i}} M \\
= & \left\|x_{n}-\frac{\sum_{i=1}^{n} \gamma_{i} t_{i} x_{n}}{\sum_{i=1}^{n} \gamma_{i}}\right\|+\frac{2 \sum_{i=n+1}^{\infty} \gamma_{i}}{\sum_{i=1}^{\infty} \gamma_{i}} M \\
\leq & \left\|x_{n}-\frac{\sum_{i=1}^{n} \gamma_{i} t_{i} y_{n}}{\sum_{i=1}^{n} \gamma_{i}}\right\|+\left\|\frac{\sum_{i=1}^{n} \gamma_{i} t_{i} y_{n}}{\sum_{i=1}^{n} \gamma_{i}}-\frac{\sum_{i=1}^{n} \gamma_{i} t_{i} x_{n}}{\sum_{i=1}^{n} \gamma_{i}}\right\| \\
& +\frac{2 \sum_{i=n+1}^{\infty} \gamma_{i}}{\sum_{i=1}^{\infty} \gamma_{i}} M .
\end{aligned}
$$

From Step 2 and (3.3), we obtain $\lim _{n \rightarrow \infty}\left\|x_{n}-\frac{\sum_{i=1}^{\infty} \gamma_{i} t_{i} x_{n}}{\sum_{i=1}^{\infty} \gamma_{i}}\right\|=0$.

Step 5. $\lim _{n \rightarrow \infty} x_{n}=v \in F$ :

Define a mapping $t: E \rightarrow E$ by

$$
t x=\frac{\sum_{n=1}^{\infty} \gamma_{n} t_{n} x}{\sum_{n=1}^{\infty} \gamma_{n}}
$$

for any $x \in E$. By Lemma 2.2, $t$ is well defined, nonexpansive and $\operatorname{Fix}(t)=\bigcap_{n=1}^{\infty} \operatorname{Fix}\left(t_{n}\right)$. Since $E$ is compact, there exists a subsequence $\left\{x_{n_{k}}\right\}$ of $\left\{x_{n}\right\}$ which converges to $v$ for some $v \in E$. Using Step 3 and Step 4, we have

$$
\begin{aligned}
\|t v-v\| & \leq \lim _{k \rightarrow \infty}\left(\left\|t v-t x_{n_{k}}\right\|+\left\|t x_{n_{k}}-x_{n_{k}}\right\|+\left\|x_{n_{k}}-v\right\|\right) \\
& \leq \lim _{k \rightarrow \infty}\left(\left\|t x_{n_{k}}-x_{n_{k}}\right\|+2\left\|x_{n_{k}}-v\right\|\right)=0
\end{aligned}
$$


and

$$
\begin{aligned}
\operatorname{dist}(v, T v) & \leq\left\|v-x_{n_{k}}\right\|+\operatorname{dist}\left(x_{n_{k}}, T x_{n_{k}}\right)+H\left(T x_{n_{k}}, T v\right) \\
& \leq\left\|v-x_{n_{k}}\right\|+\left\|x_{n_{k}}-z_{n_{k}}\right\|+\left\|x_{n_{k}}-v\right\| \rightarrow 0 \quad \text { as } k \rightarrow \infty
\end{aligned}
$$

It follows that $v \in \operatorname{Fix}(T) \cap \operatorname{Fix}(t)=F$. Since $\lim _{n \rightarrow \infty}\left\|x_{n}-v\right\|$ exists by Step $1, \lim _{n \rightarrow \infty} \| x_{n}-$ $v\left\|=\lim _{k \rightarrow \infty}\right\| x_{n_{k}}-v \|=0$.

The following example shows that the condition ' $T w=\{w\}$ for all $w \in F$ ' in Theorem 3.2 is necessary.

Example 3.3 We consider the space $X$ of Example 3.9 in [8]. Let $X$ be the Hilbert space $\mathbb{R}^{2}$ with the usual norm, and let $f:[0,1] \rightarrow[0,1]$ be a continuous strictly concave function such that $f(0)=\frac{1}{2}, f(1)=1$ and $f^{\prime}(x) \leq 1$ for all $x \in[0,1]$. Let $\varepsilon_{n}=\sum_{i=1}^{n}\left(\frac{1}{2}\right)^{i+1}, T:[0,1]^{2} \rightarrow$ $F B\left([0,1]^{2}\right)$ be defined by $T(a, b)=[0,1] \times[f(a), 1]$ and $t_{n}:[0,1]^{2} \rightarrow[0,1]^{2}$ be defined by

$$
t_{n}(a, b)= \begin{cases}\left(a, \varepsilon_{n}\right), & b<\varepsilon_{n} \\ (a, b), & \text { otherwise }\end{cases}
$$

It is straightforward showing that $T$ and each $t_{n}$ are nonexpansive. Set $x_{1}=(1,0) \in[0,1]^{2}$ and for a subsequence $\left\{\gamma_{n}\right\}$ in $(0,1)$ with $\sum_{n=1}^{\infty} \gamma_{n}<1$. Let $\left\{x_{n}=\left(a_{n}, b_{n}\right)\right\}$ be a sequence in $[0,1]^{2}$ defined as

$$
\begin{aligned}
& y_{n}=\frac{1}{2} x_{n}+\frac{1}{2} z_{n}, \\
& x_{n+1}=\left(1-\sum_{i=1}^{n} \gamma_{i}\right) x_{n}+\sum_{i=1}^{n} \gamma_{i} t_{i} y_{n},
\end{aligned}
$$

where

$$
z_{n}= \begin{cases}\left(0, f\left(a_{n}\right)\right), & n \text { is odd } \\ \left(1, f\left(a_{n}\right)\right), & n \text { is even. }\end{cases}
$$

We will show that $\left\{x_{n}\right\}$ does not converge to a common fixed point of $T$ and $\left\{t_{n}\right\}$.

Proof Clearly, $\left\{z_{n}\right\}$ is a divergent sequence. We note that $\varepsilon_{n} \uparrow \frac{1}{2}$ and for each $y=(a, b) \in$ $[0,1]^{2}$ with $b \geq \frac{1}{2}$, we have $t_{i} y=y$ for all $i$. If we put $y_{n}=\left(c_{n}, d_{n}\right)$, then $d_{n} \geq \frac{1}{2}$ for all $n$. Since $\sum_{n=1}^{\infty} \gamma_{n}<1$, we must have $d\left(x_{n}, z_{n}\right) \rightarrow 0$ as $n \rightarrow \infty$. Suppose $\left\{x_{n}\right\}$ converges to $z$ for some $z \in F=\left\{(a, b) \in[0,1]^{2}: b \geq f(a)\right\}$. Thus $\left\{z_{n}\right\}$ also converges to $z$, a contradiction.

It is noticed that $F$ is not convex. Thus it is not a nonexpansive retract of any convex set. It can be also observed that if we redefine the mapping $T$ as $T(a, b)=\{a\} \times\left[\frac{1+b}{2}, 1\right]$ we can easily verify that $T$ is nonexpansive and the condition (2.1) is satisfied. 
Remark 3.4 With the same proof, Theorem 3.2 is valid when $\left\{x_{n}\right\}$ is of the following form: For a permutation $\pi$ on $\mathbb{N}$, define $\left\{x_{n}\right\}$ in $E$ by $x_{1} \in E$ and

$$
\begin{aligned}
& y_{n}=\left(1-\beta_{n}\right) x_{n}+\beta_{n} z_{n}, \\
& x_{n+1}=\left(1-\sum_{i=1}^{n} \gamma_{\pi(i)}\right) x_{n}+\sum_{i=1}^{n} \gamma_{\pi(i)} t_{\pi(i)} y_{n},
\end{aligned}
$$

$z_{n} \in T x_{n}$, and $0<a \leq \beta_{n} \leq b<1$.

Note also that the above result is equivalent to:

Let $\left\{I_{n}\right\}$ be a sequence of subsets of $\mathbb{N}$ satisfying $I_{n} \subset I_{n+1}$ for $n \in \mathbb{N}$ and $\bigcup_{n=1}^{\infty} I_{n}=\mathbb{N}$. Define $\left\{x_{n}\right\}$ in $E$ by $x_{1} \in E$ and

$$
\begin{aligned}
& y_{n}=\left(1-\beta_{n}\right) x_{n}+\beta_{n} z_{n}, \\
& x_{n+1}=\left(1-\sum_{i \in I_{n}} \gamma_{i}\right) x_{n}+\sum_{i \in I_{n}} \gamma_{i} t_{i} y_{n},
\end{aligned}
$$

$z_{n} \in T x_{n}$, and $0<a \leq \beta_{n} \leq b<1$. Then the sequence $\left\{x_{n}\right\}$ converges strongly to some $v \in F$.

Thus Theorem 3.2 contains Theorem 1.3.

With the application of the demiclosedness principle (Theorem 2.1), a weak convergence version of Theorem 3.2 also holds:

Theorem 3.5 Let $X$ be a strictly convex Banach space satisfying the Opial's condition, $E$ be a weakly compact convex subset of $X$, let $\left\{t_{n}: n \in \mathbb{N}\right\}$ be a family of single-valued nonexpansive mappings on $E$, and let $T: E \rightarrow F B(E)$ be a multivalued nonexpansive mapping. Suppose $F \neq \emptyset$ and $T w=\{w\}$ for all $w \in F$. Given a sequence of positive numbers $\left\{\gamma_{n}\right\}$ with $0<\sum_{n=1}^{\infty} \gamma_{n}<1$ and $\left\{\beta_{n}\right\}$ with $0<a \leq \beta_{n} \leq b<1$. Then the sequence $\left\{x_{n}\right\}$ defined by (3.1) converges weakly to some $v \in F$.

Proof In the proof of Theorem 3.2, by applying the Opial's condition, it follows from a standard argument that $\left\{x_{n}\right\}$ converges weakly to some $v \in E$. Then Theorem 2.1 implies that $v$ is a point in $F$.

\subsection{CAT(0) spaces}

Let $E$ be a nonempty bounded closed convex subset of a complete CAT(0) space $X$, let $\left\{t_{n}: n \in \mathbb{N}\right\}$ be a family of single-valued nonexpansive mappings on $E$, and $T: E \rightarrow F B(E)$ be a multivalued nonexpansive mapping. Given $\left\{\gamma_{n}\right\}$ a sequence of positive numbers with $\sum_{n=1}^{\infty} \gamma_{n}<1$ and $\sum_{i=n}^{\infty} \gamma_{i}^{\prime} \rightarrow 0$ as $n \rightarrow \infty$ where $\gamma_{n}^{\prime}=\sum_{i=n+1}^{\infty} \gamma_{i}$. The sequence $\left\{x_{n}\right\}$ of the modified Ishikawa iteration is defined by

$$
\begin{aligned}
& y_{n}=\left(1-\beta_{n}\right) x_{n} \oplus \beta_{n} z_{n}, \\
& x_{n+1}=\left(1-\sum_{i=1}^{n} \gamma_{i}\right) x_{n} \oplus\left(\sum_{i=1}^{n} \gamma_{i}\right) \bigoplus_{i=1}^{n} \frac{\gamma_{i}}{\sum_{i=1}^{n} \gamma_{i}} t_{i} y_{n},
\end{aligned}
$$

where $x_{1} \in E, z_{n} \in T x_{n}$, and $0<a \leq \beta_{n} \leq b<1$. Put $F:=\bigcap_{n=1}^{\infty} \operatorname{Fix}\left(t_{n}\right) \cap \operatorname{Fix}(T)$. 
Theorem 3.6 Let $E$ be a compact convex subset of a complete $\mathrm{CAT}(0)$ space $X$. Let $\left\{t_{n}: n \in\right.$ $\mathbb{N}\}$ be a family of single-valued nonexpansive mappings on $E$, and let $T: E \rightarrow F B(E)$ be a multivalued nonexpansive mapping. Suppose $F \neq \emptyset$ and $T w=\{w\}$ for all $w \in F$. Given $\left\{\gamma_{n}\right\}$ a sequence of positive numbers with $\sum_{n=1}^{\infty} \gamma_{n}<1$ and $\sum_{i=n}^{\infty} \gamma_{i}^{\prime} \rightarrow 0$ as $n \rightarrow \infty$ where $\gamma_{n}^{\prime}=$ $\sum_{i=n+1}^{\infty} \gamma_{i}$. If $0<a \leq \beta_{n} \leq b<1$, then the sequence $\left\{x_{n}\right\}$ defined by (3.5) converges strongly to some $v \in F$.

Proof The proof follows along the lines with the proof of Theorem 3.2. Recall that $w_{1} x=$ $t_{1} x$ and $w_{n} x=\bigoplus_{i=1}^{n} \frac{\gamma_{i}}{\sum_{i=1}^{n} \gamma_{i}} t_{i} x$ for all $n \geq 2$. Thus, by (3.5),

$$
x_{n+1}=\left(1-\sum_{i=1}^{n} \gamma_{i}\right) x_{n} \oplus\left(\sum_{i=1}^{n} \gamma_{i}\right) w_{n} y_{n} .
$$

As before, we consider the proof in 5 steps. Because of the same details in some cases, we only present proofs for Step 2 to Step 4 .

Step 2. $\lim _{n \rightarrow \infty} d\left(x_{n}, w_{n} y_{n}\right)=0$ :

Let $w \in F$, we have $w_{n} w=w$ for all $n$. Using the nonexpansiveness of $w_{n}$, we see that

$$
d\left(w_{n} y_{n}, w\right) \leq d\left(y_{n}, w\right) \leq\left(1-\beta_{n}\right) d\left(x_{n}, w\right)+\beta_{n} d\left(z_{n}, w\right) \leq d\left(x_{n}, w\right) .
$$

By (3.6) and using $(\mathrm{CN})$ inequality,

$$
\begin{aligned}
d^{2}\left(x_{n+1}, w\right) \leq & \left(1-\sum_{i=1}^{n} \gamma_{i}\right) d^{2}\left(x_{n}, w\right)+\left(\sum_{i=1}^{n} \gamma_{i}\right) d^{2}\left(w_{n} y_{n}, w\right) \\
& -\sum_{i=1}^{n} \gamma_{i}\left(1-\sum_{i=1}^{n} \gamma_{i}\right) d^{2}\left(x_{n}, w_{n} y_{n}\right) \\
\leq & d^{2}\left(x_{n}, w\right)-\sum_{i=1}^{n} \gamma_{i}\left(1-\sum_{i=1}^{n} \gamma_{i}\right) d^{2}\left(x_{n}, w_{n} y_{n}\right) .
\end{aligned}
$$

Let $\gamma=\sum_{i=1}^{\infty} \gamma_{i}$. Since $0<\gamma_{1} \leq \sum_{i=1}^{n} \gamma_{i} \leq \gamma<1$,

$$
\gamma_{1}(1-\gamma) d^{2}\left(x_{n}, w_{n} y_{n}\right) \leq \sum_{i=1}^{n} \gamma_{i}\left(1-\sum_{i=1}^{n} \gamma_{i}\right) d^{2}\left(x_{n}, w_{n} y_{n}\right) \leq d^{2}\left(x_{n}, w\right)-d^{2}\left(x_{n+1}, w\right) .
$$

This implies that

$$
\sum_{n=1}^{\infty}\left[\gamma_{1}(1-\gamma) d^{2}\left(x_{n}, w_{n} y_{n}\right)\right] \leq d^{2}\left(x_{1}, w\right)<\infty
$$

and hence $\lim _{n \rightarrow \infty} d\left(x_{n}, w_{n} y_{n}\right)=0$.

Step 3. $\lim _{n \rightarrow \infty} d\left(x_{n}, z_{n}\right)=0$ :

Using (3.6) and (CN) inequality, we have

$$
\begin{aligned}
d^{2}\left(w_{n} y_{n}, w\right) & \leq d^{2}\left(y_{n}, w\right) \leq\left(1-\beta_{n}\right) d^{2}\left(x_{n}, w\right)+\beta_{n} d^{2}\left(z_{n}, w\right)-\beta_{n}\left(1-\beta_{n}\right) d^{2}\left(x_{n}, z_{n}\right) \\
& \leq d^{2}\left(x_{n}, w\right)-\beta_{n}\left(1-\beta_{n}\right) d^{2}\left(x_{n}, z_{n}\right),
\end{aligned}
$$


and thus

$$
\begin{aligned}
d^{2}\left(x_{n+1}, u\right) & \leq\left(1-\sum_{i=1}^{n} \gamma_{i}\right) d^{2}\left(x_{n}, w\right)+\sum_{i=1}^{n} \gamma_{i} d^{2}\left(w_{n} y_{n}, w\right) \\
& \leq d^{2}\left(x_{n}, w\right)-\beta_{n}\left(\sum_{i=1}^{n} \gamma_{i}\right)\left(1-\beta_{n}\right) d^{2}\left(x_{n}, z_{n}\right) .
\end{aligned}
$$

As before,

$$
a \gamma_{1}(1-b) d^{2}\left(x_{n}, z_{n}\right) \leq \beta_{n}\left(\sum_{i=1}^{n} \gamma_{i}\right)\left(1-\beta_{n}\right) d^{2}\left(x_{n}, z_{n}\right) \leq d^{2}\left(x_{n}, w\right)-d^{2}\left(x_{n+1}, w\right) .
$$

This also implies that $\lim _{n \rightarrow \infty} d\left(x_{n}, z_{n}\right)=0$.

Step 4. $\lim _{n \rightarrow \infty} d\left(x_{n}, t x_{n}\right)=0$, where $t=\bigoplus_{i=1}^{\infty} \frac{\gamma_{i}}{\sum_{i=1}^{\infty} \gamma_{i}} t_{i}$ :

Since $E$ is compact, there exists a subsequence $\left\{y_{n^{\prime}}\right\}$ of $\left\{y_{n}\right\}$ such that $y_{n^{\prime}} \rightarrow y$ as $n^{\prime} \rightarrow \infty$ for some $y \in E$. Using the nonexpansiveness of $w_{n^{\prime}}$ and $t$, we have

$$
\begin{aligned}
d\left(w_{n^{\prime}} y_{n^{\prime}}, t y_{n^{\prime}}\right) & \leq d\left(w_{n^{\prime}} y_{n^{\prime}}, w_{n^{\prime}} y\right)+d\left(w_{n^{\prime}} y, t y\right)+d\left(t y, t y_{n^{\prime}}\right) \\
& \leq 2 d\left(y_{n^{\prime}}, y\right)+d\left(w_{n^{\prime}} y, t y\right) \rightarrow 0 \quad \text { as } n^{\prime} \rightarrow \infty .
\end{aligned}
$$

Therefore, $\lim _{n \rightarrow \infty} d\left(w_{n} y_{n}, t y_{n}\right)=0$. From Step 2 and Step 3 we have

$$
\begin{aligned}
d\left(x_{n}, t x_{n}\right) & \leq d\left(x_{n}, t y_{n}\right)+d\left(t y_{n}, t x_{n}\right) \\
& \leq d\left(x_{n}, t y_{n}\right)+d\left(y_{n}, x_{n}\right) \\
& \leq d\left(x_{n}, w_{n} y_{n}\right)+d\left(w_{n} y_{n}, t y_{n}\right)+\beta_{n} d\left(x_{n}, z_{n}\right) \rightarrow 0 \quad \text { as } n \rightarrow \infty .
\end{aligned}
$$

\section{Competing interests}

The authors declare that they have no competing interests.

\section{Authors' contributions}

All authors read and approved the final manuscript.

\section{Acknowledgements}

The authors are grateful to the referees for their valuable comments and suggestions. They also would like to thank the Junior Science Talent Project (JSTP) under Thailand's National Science and Technology Development Agency for financial support.

Received: 17 March 2012 Accepted: 28 June 2012 Published: 19 July 2012

\section{References}

1. Belluce, LP, Kirk, WA: Fixed-point theorems for families of contraction mappings. Pac. J. Math. 18, 213-217 (1966)

2. Belluce, LP, Kirk, WA: Nonexpansive mappings and fixed-points in Banach spaces. III. J. Math. 11, 474-479 (1967)

3. Bridson, M, Haefliger, A: Metric Spaces of Non-positive Curvature. Springer, Berlin (1999)

4. Browder, FE: Nonexpansive nonlinear operators in a Banach space. Proc. Natl. Acad. Sci. USA 54, 1041-1044 (1965)

5. Bruck, RE: Properties of common fixed-point sets of nonexpansive mappings in Banach spaces. Trans. Am. Math. Soc. 179, 251-262 (1973)

6. Bruck, RE: A common fixed point theorem for a commuting family of nonexpansive mappings. Pac. J. Math. 53, 59-71 (1974)

7. Butsan, T, Dhompongsa, S, Fupinwong, W: Schauder's conjecture and the Kakutani fixed point theorem on convex metric spaces. J. Nonlinear Convex Anal. 11, 513-526 (2010)

8. Dhompongsa, S, Nanan, N: A common fixed points for commuting family of single valued nonexpansive mappings and a multivalued nonexpansive mapping. Fixed Point Theory Appl. 2011, 54 (2011)

9. Dhompongsa, S, Kaewkhao, A, Panyanak, B: On Kirk's strong convergence theorem for multivalued nonexpansive mappings on CAT(0) spaces. Nonlinear Anal. 75, 459-468 (2012) 
10. Dozo, EL: Multivalued nonexpansive mappings and Opial's condition. Proc. Am. Math. Soc. 38, $286-292$ (1973)

11. Holmes, RD, Lau, AT-M: Non-expansive actions of topological semigroups and fixed points. J. Lond. Math. Soc. 5 330-336 (1972)

12. Goebel, K, Kirk, WA: Topics in Metric Fixed Point Theory. Cambridge University Press, Cambridge (1990)

13. Lau, AT-M: Normal structure and common fixed point properties for semigroups of nonexpansive mappings in Banach spaces. Fixed Point Theory Appl. 2010, Article ID 580956 (2010)

14. Lim, TC: Characterizations of normal structure. Proc. Am. Math. Soc. 43, 313-319 (1974)

15. Reich, S, Sheman, L: Two algorithms for nonexpansive mappings. Fixed Point Theory 12(2), 443-448 (2011)

16. Schu, J: Weak and strong convergence to fixed points of asymptotically nonexpansive mappings. Bull. Aust. Math. Soc. 43, 153-159 (1991)

17. Sokhuma, K, Kaewkhao, A: Ishikawa iterative process for a pair of single-valued and multivalued nonexpansive mappings in Banach spaces. Fixed Point Theory Appl. 2011, Article ID 618767 (2011)

18. Suzuki, T: Convergence theorems to common fixed points for infinite families of nonexpansive mappings in strictly convex Banach spaces. Nihonkai Math. J. 14, 43-54 (2003)

doi:10.1186/1687-1812-2012-112

Cite this article as: Anakkamatee and Dhompongsa: An approximation of a common fixed point of nonexpansive mappings on convex metric spaces. Fixed Point Theory and Applications 2012 2012:112.

\section{Submit your manuscript to a SpringerOpen ${ }^{\circ}$ journal and benefit from:}

- Convenient online submission

- Rigorous peer review

- Immediate publication on acceptance

Open access: articles freely available online

- High visibility within the field

- Retaining the copyright to your article 